\title{
The evolution of Western tonality: a corpus analysis of 24,000 songs from 190 composers over six centuries
}

Pu Huang ${ }^{1}$, Mark Wilson $^{1}$, Dustin Mayfield-Jones ${ }^{1,2}$, Viktoriya Coneva ${ }^{1,3}$, Margaret Frank ${ }^{1}$, Daniel H. Chitwood ${ }^{1,4,5,6, \S}$

${ }^{1}$ Donald Danforth Plant Science Center, St. Louis, MO 63132

${ }^{2}$ Current address: Department of Plant Biology, Carl R. Woese Institute for Genomic Biology, University of Illinois at Urbana-Champaign, Urbana, IL 61801

${ }^{3}$ Current address: Department of Biology, Kenyon College, Gambier, OH 43022

${ }^{4}$ Independent Researcher, Santa Rosa, CA 95409

${ }^{5}$ Current address: Department of Horticulture, Michigan State University, East Lansing, MI 48824

${ }^{6}$ Current address: Computational Mathematics, Science and Engineering, Michigan State University, East Lansing, MI 48824

$\S$ To whom correspondence should be addressed:

Daniel H. Chitwood

Departments of Horticulture and Computational Mathematics, Science and Engineering Michigan State University

East Lansing, MI 48824

email: chitwoo9@msu.edu 


\begin{abstract}
The corpus of Western music offers the chance to analyze trends in its evolution. Here, we analyze greater than 24,000 MIDI (Musical Instrument Digital Interface) transcriptions of Western classical music from 197 composers spanning from the $15^{\text {th }}$ to $20^{\text {th }}$ centuries. The unique file format of MIDI files (notes of discrete frequencies turning "on" and "off" at specific times) allows us to statistically quantify note usage with respect to pitch class and intervals. We first perform a Principal Component Analysis (PCA) on pitch class and show that the data creates a ring. Songs strongly associated with a particular key fall on the ring, whereas highly chromatic and atonal works fall in the center, revealing that the evolution of Western music is constrained by the circle of fifths. We then examine interval usage. Discriminant analysis on composer identity reveals that a major source of evolutionary change is incremental and linear. Interval usage predicts individual composer identity at levels above chance. A Self-Organizing Map (SOM) reveals four major groups of composers with similar tonal styles. These groups are loosely based on traditional musical eras, but reveal unexpected continuity between co-existing schools of composers.
\end{abstract}

\title{
Introduction
}

Music is an especially mathematical cultural medium. The notes of scales are related to each other as Pythagorean ratios and through harmonic series [1]. Voice leading and counterpoint describe linear progressions of melodic lines and their interactions with each other to produce pleasing harmonies [2]. Chord relationships can be represented as a continuous ring (the circle of fifths) [3,4], a two-dimensional lattice (the Tonnetz) [5], and geometrically in neoRiemannian and other non-Euclidian spaces $[6,7]$. Rhythm, too, is a form of mathematical complexity in music $[8,9]$.

Separate from the mathematical theory of music, the discrete nature of music, using a finite number of pitches that are temporally arranged, permits statistical analyses. The ability to notate music, and a historical record, allows a corpus analysis of cultural trends. Just as similar analyses of written texts analyzing word frequency across centuries and geographies reveals the evolution of language on a macro scale [10], music too can be reduced to count data and its evolution studied. Large corpuses of both recordings and music notation formats provide datasets to study historical trends [11,12]. A number of machine readable music notation formats (MIDI and MusicXML, among many others) $[13,14]$ enable data analysis using computation [15-19]. These resources have led to insights into the evolution of music analyzing pitch, timbre, loudness, and harmonics, especially for popular music, which has a rich corpus to sample from over recent history, from its inception to the present $[20,21]$.

Here, we analyze the tonality of $>24,000$ MIDI (Musical Instrument Digital Interface) files, representing songs from 197 composers from the $15^{\text {th }}$ to $20^{\text {th }}$ centuries. Pitch class and interval usage is tallied and scaled for each song. A Principal Component Analysis (PCA) on pitch class reveals that the circle of fifths is more than a metaphor: the evolution of tonal variance in Western music is constrained to a ring, where tonal songs reside on the edges and atonal and 
chromatic pieces are in the center. Songs from composers shift along the ring historically, as more complicated key signatures with more accidentals are used later in history. Intervals, arising from both simultaneous and sequential notes, also strongly vary across history. A Linear Discriminant Analysis (LDA) on composer identity reveals that incremental, linear changes in interval use define the history of Western music. A self-Organizing Map (SOM) reveals four main groups of composers with similar tonal styles. Unlike the conventionally defined eras in music history, the groups show considerable historical overlap, suggesting co-existing schools of tonality across the evolution of Western tonality.

\section{Results}

Western music is constrained along the circle of fifths

For >24,000 MIDI (Musical Instrument Digital Interface) files, we first analyzed the frequency of pitch class usage. Each MIDI file was divided into "bins" (Fig. 1). We created the bin concept to facilitate the analysis of intervals (see below) but used it first to analyze pitch class. A bin is an assembly of notes that is temporally defined by either a "note on" or "note off" message in the MIDI file (that is, the temporal beginning or end of any note). Therefore, for every note change a new bin is defined. Each bin is an assembly of notes that are simultaneously on during a period in the music. Because a bin is defined by a note change, it is independent of tempo or meter, an important consideration when comparing diverse songs. To measure pitch class usage, the MIDI note numbers $0-127$, where 0 corresponds to $C$ and middle $C$ is 60 (Fig. 1B), were converted to modulus 12 (Fig. 1C). By using modulus 12 , note values 0-127 are converted to pitch classes $0-11$, in which $0=C, 1=C \#, 2=D, 3=D \#, 4=E, 5=F, 6=F \#, 7=G, 8=G \#, 9=A, 10=A \#$, and $11=\mathrm{B}$. These values represent pitch class regardless of the octave of the note.

For each MIDI file, the pitch class of each note from each bin is tallied. The result is a count of the notes within each bin, for pitch classes 0-11, representing their frequency in the MIDI file. The count of pitch classes $0-11$ is then scaled, so that the mean across pitch classes is 0 and the variance 1 . A negative scaled pitch class value therefore indicates an under-representation of that pitch class relative to others for a given MIDI file (and a positive value an over representation). The scaled pitch class values are comparable across MIDI files as an indicator of pitch class usage (regardless of the number of notes in a MIDI file).

We performed a Principal Component Analysis (PCA) on scaled pitch class values for $>24,000$ MIDI files. If PC2 (25.7\%) is plotted against PC1 (37.9\%) a ring, with discrete densities, is seen (Fig. 2A). Because scaled pitch class values represent a summary of pitch class usage across each MIDI file, we hypothesized the discrete densities might correspond to major and minor keys. To test this idea, we projected scaled pitch values corresponding to major (black) and minor (blue) triads from each key onto the PCA space (Fig. 2A). The circle of fifths, a succession of keys related to each other by a fifth (i.e., seven half steps) is recapitulated by the projected triads. For example, for major keys, a density corresponding to the $\mathrm{C}$ major triad is next to $\mathrm{G}$, which is next to $D$, which is next to $A$ and so on, each a fifth higher than the last until the circle comes back to $\mathrm{C}$. A similar trend is seen for minor keys, in which the relative minor of $\mathrm{C}$ major, a 


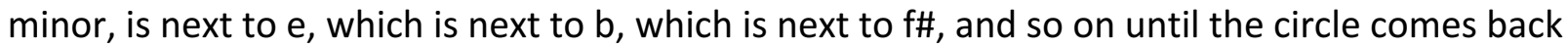
to a. We note that the relative major and minor keys (e.g., $\mathrm{C}$ major and a minor) are shifted one domain from each other. From this data, we conclude that -statistically-the circle of fifths, which is the foundation of Western tonality, is not a metaphor, but is in fact literally a circle of related keys. A majority of the variance describing key (PC1 and PC2, 63.6\%) across Western music history resides along this ring, and the evolution of tonal music is constrained by it.

To further dissect the tonal representation of the PCA based on pitch class usage, we next projected intervals onto the PCA space. Each pairwise combination of pitch classes was converted into scaled pitch class values. Each pair of pitch classes is first represented by a letter indicating the lower pitch class followed by a number, which is the number of half steps the higher pitch class is above the lower (Fig. 2B). The projected intervals form concentric rings in the PCA corresponding to inverted interval pairs. Inverted interval pairs are defined by transposing one member of an interval an octave above the other. For the example of a fifth where $C$ is the lower note and $G$ the upper, if $C$ is transposed an octave above $G$, the resulting interval (where $G$ is now the lower note) is a fourth. The inverted interval pair of a perfect fifth and perfect fourth (P5/P4; light blue/dark blue) forms an outer ring in the PCA space (Fig. 2B). Minor seventh and major second (m7/M2; light green/dark green), major sixth and minor third (M6/m3; yellow/light orange), minor sixth and major third (m6/M3; pink/red), and major seventh and minor second (M7/m2; lavender/purple) inverted interval pairs form nested concentric rings. Perfect unison (P1; orange) intervals form a ring in the middle of other inverted interval pairs and diminished fifths ( $d 5$; black) are found in the middle of the ring. We note that commonly used intervals (P5/P4) are found on the outside of the ring, where most the density of the data is also found, whereas rarely used intervals such as half steps (M7/m2) and diminished fifths (d5) are found in the center of the ring. We revisit the idea of frequent and rare intervals residing on the outside and in the center of the ring when discussing historical trends in the data later.

We projected scaled pitch class values for triads (Fig. 2A) and intervals (Fig. 2B) onto the PCA space to find densities corresponding to key. A more empirical measure would be to use the known key of compositions. To do so, we used the assigned keys of compositions from the most well-represented composer in our dataset, Johann Sebastian Bach, using associated BWV numbers in MIDI files. Both major (Fig. 2C) and minor (Fig. 2D) BWV compositions correspond to the location in the PCA space of major and minor triads (Fig. 2A), although the BWV data is found on the outer edge of the ring where most the data lies, compared to triads. To understand the pitch class signature of BWV compositions, we visualized the aggregated scaled pitch class values of BWV compositions by key (Fig. 2E-F). Pitch class usage is what is expected from music theory. For major BWV pieces the tonic (in C major, $\mathrm{C}$ ) and fifth (in $\mathrm{C}$ major, $\mathrm{G}$ ) are over-represented, while the minor second (in $\mathrm{C}$ major, $\mathrm{C \#}$ ) and minor third (in C major, D\#) are under-represented (Fig. 2E). For minor BWV pieces, similar over-representation of the tonic and fifth is observed as well as under-representation of the minor second, but it is the major third (in c minor, E) rather than the minor third that is under-represented, and the diminished fifth (in c minor, F\#) is also more under-represented compared to major keys (Fig. 2F). 


\section{Shifts in key and interval usage across history}

Associated with each MIDI file is not only key, but a composer who wrote the song. We projected the year of death of composers for each MIDI file onto the PCA (Fig. 3A). Composer years of death span 1453 to 1992. Composers that died early in history tend to have higher PC2 values, associated with key signatures with few accidentals (such as C, G, and F major, see Fig. 2A). Few songs have key signatures with a higher number of accidentals (like C\#, F\#, and B major), or reside in the center of the ring where seldom used intervals, like minor seconds or diminished fifths (see Fig. 2B for reference), reside. These songs, however, tend to come from later in history (Fig. 3A). To explore this observation, we projected well-known composers from throughout history with distinct tonalities onto the PCA space (Fig. 3B). Highly tonal early music and Baroque composers, like Giovanni Pierluigi da Palestrina (c. 1525-1594) and Antonio Vivaldi (1678-1741), tend to compose in key signatures with few accidentals, and their compositions occupy the edges of the ring (Fig. 3B). Composers from later in history that extensively used chromaticism and avoided tonal centers, like Richard Wagner (1813-1883) and Dmitri Shostakovich (1906-1975), are more evenly distributed throughout the PCA space, in both regions associated with key signatures that have many accidentals and the inside the ring. Compositions from Arnold Schoenberg (1874-1951), who practiced atonality, tend to be found inside the ring, rather than on the edge of the ring where more tonal compositions (which are the majority) reside (Fig. 3B).

We wondered if interval usage might show similar historical shifts as pitch class usage. To measure interval usage, we again used "bins", assemblies of notes that are temporally defined by "note on" and "note off" events that are independent of tempo and time signature (Fig. 1). Interval usage was measured in two ways: "within" and "between" bins. The "within" bin analysis looks at each pairwise combination of intervals within each bin for a MIDI file (similar to chords, but defined by bin). Each interval is recorded as the difference in half-steps and a modulus 12 applied, such that 0 is perfect unison and 11 a major seventh, regardless if the interval is greater than an octave. A count of all pairwise intervals used within each bin across the bins of a song is taken and scaled. The "between" bin analysis is done similarly as the "within" bin analysis, except that instead of taking all pairwise intervals within a bin, all pairwise intervals between successive bins are taken. The result is that the "within" bin analysis reflects notes concurrently played whereas the "between" bin analysis is more dynamic, representing changes in notes between bins. A PCA performed on both the "within" (Fig. 4A) and the "between" (Fig. 4B) bin analyses shows distinct separation of compositions from early and late in history, indicating historical shifts in interval usage and tonality.

\section{Linear, historical changes in the tonality underlie its evolution}

Historical changes in interval usage (Fig. 4) imply differences between composers as well. To investigate the ability of intervals to discriminate composers, we performed a linear discriminant analysis (LDA) for composer identity based on "within" and "between" bin analyses (Fig. 5). The first linear discriminant (LD), that explains $14.2 \%$ of differences between composers (Fig. 5A), is uniquely and highly correlated with composer year of death (Fig. 5B; $\mathbf{B H}$ - 
adjusted $\mathrm{p}$ value $=1.55 \times 10^{-48}$, rho $=0.82$ ). The correlation between LD1 with year of death is roughly linear (Fig. 5C-D). The results indicate that the linear progression of history, alone, is one of the main determinants of tonality among composers. This axis of variation (LD1, 14.2\%) has less to do with any individualistic contribution of the composer to the tonal composition of their songs, and more with simply where in history, by chance, a composer was born. This scenario is identical to the case of violin morphology where, again, the primary axis of variation differentiating luthiers is the first discriminant, and highly correlated with history in a linear fashion [22]. Such results indicate that time, alone, is a major contributor to cultural evolution.

\section{Predicting composer identity}

To identify groups of composers with similar tonal usage, we performed a Self-Organizing Map (SOM) analysis on the linear discriminants. Using SOMs, four main groups of composers were identified. The earliest group (Fig. 5D, orange) includes not only Franco-Flemish composers of the Early Renaissance, such as Guillaume Du Fay (c. 1397-1474), Johannes Ockeghem (1410/1425-1497), Jacob Obrecht (1457/8-1505), Heinrich Isaac (c. 1450-1517), and Josquin des Prez (c. 1450/1455-1521), but extends into the Early Baroque period and beyond with composers such as Johann Jakob Froberger (1616-1667), Jean-Baptiste Lully (1632-1687), Dieterich Buxtehude (c. 1637/39-1707), Arcangelo Corelli (1653-1713), and notably, Francis Poulenc (1899-1963). The next early group typifies the Renaissance period (Fig. 5D, magenta), including Giovanni Pierluigi da Palestrina (c. 1525-1594), Tomás Luis de Victoria (c. 1548-1611), William Byrd (c. 1539/40 or 1543-1623), John Dowland (1563-1626), and Claudio Monteverdi (1567-1643). The Baroque to Modern group (Fig. 5D, lavender) spans a large swath of history and diverse musical styles, with some of the most prolific and popular composers in history, including Johann Sebastian Bach (1685-1750), Wolfgang Amadeus Mozart (1756-1791), Ludwig van Beethoven (1770-1827), Igor Stravinsky (1882-1971), and Dmitri Shostakovich (1906-1975). The final group is comprised of a single composer: Arnold Schoenberg (1874-1951) (Fig. 5D, green). That Schoenberg's tonality is an outlier and clusters by itself is likely because of his use of atonality, falling outside the circle of fifths (see Fig. 3B).

We then determined the degree to which tonality can predict composer identity. Results from an LDA to discriminate composer identity using "within" and "between" bin analyses are represented as a confusion matrix (Fig. 6), in which actual composer identity (vertical axis) is plotted against predicted composer identity (horizontal axis). A cross-validated "leave one out" approach was used, in which each song in turn is omitted, and the LDA performed without it, and the song assigned to a predicted composer using the discriminants from the analysis it was omitted. The proportions of assigned predicted composers for each row sum to one for a given actual composer, and proportions falling along the diagonal represent correct predictions. The overall correct prediction rate is $10.2 \%$. Although seemingly small, the prediction is distributed over 197 composers using summary statistics of tonality alone, and no other stylistic indicators. To determine the significance of the prediction, a bootstrapping approach, permuting composer identity against the "within" and "between" bin analyses 1,000 times and recalculating the correct prediction rate was performed. 0 out of 1,000 times was the permuted prediction rate greater than the actual rate of $10.2 \%$. The maximum permutated rate was 
$0.17 \%$, the mean $0.059 \%$ and the median $0.058 \%$. From this analysis, we conclude that interval frequency contains significant predictive power with respect to composer identity.

\section{Co-existing schools of composers with similar tonality across history}

Composers in the confusion matrix are arranged by their SOM groups (Fig. 6). When songs are misidentified, they tend to be assigned to composers in the same SOM group (Fig. 6), again suggesting tonal similarities between composers in each of these four groups. To determine the tonal patterns characteristic of each of these groups, we created a heat map of averaged, scaled "within" and "between" interval frequencies by composer (Fig. 7). The Renaissance group has the most striking tonal pattern, enriched especially for perfect fifths, major thirds, and perfect fourths, and depleted for diminished fifths and minor sevenths, all hallmarks of a rigid, conventional tonality. The Early Renaissance/Early Baroque group more evenly uses all intervals relative to the Renaissance. The Baroque to Modern group similarly evenly uses intervals, but is relatively depleted for perfect fifths and major thirds, the exact opposite of the tonal pattern for the Renaissance group. Schoenberg is highly enriched for major sevenths, more so than for any other interval in any other group, and is highly depleted for perfect unison, perfect fifth, minor third, and major sixth intervals.

Surprisingly, while the groups roughly correspond to conventional groupings of composers that define musical eras [23], they span large swaths of history, and extensively overlap. The most striking example is that, tonally, the Early Renaissance/Early Baroque group completely spans the Renaissance group (Fig. 5D). Similarly, J.S. Bach is in the same group as Mozart, Beethoven, Debussy, and Stravinsky, and has more in common-tonally-with these composers than Buxtehude or Lully (Fig. 5D). The contradiction with conventional groupings of composers arises from the fact that this study is viewing music history from only a single facet-summarized tonality. From this perspective, the groups make sense with respect to common interval usages, and that a single individual, Schoenberg, who practiced the absence of tonality, would alone comprise a group (Fig. 7).

\section{Discussion}

Our analysis does not account for melody, voice leading, scoring, rhythm, tempo, nuances of performance, loudness, or temporal intervals larger than successive note changes. If each of these elements were to be isolated and analyzed using similar reductionist techniques as used here, different sets of groups, comprised of different composers and trends in history would be observed [21]. However, tonality-the arrangement of pitches within music -is a fundamental attribute of music worth considering alone. Counts of notes and intervals are absolute; they do not change between performances, there is no subjective interpretation, and they are recorded in a corpus. From this perspective, analyzing tonality is not unlike analyzing word frequencies across history from digitized books [10]: structure at the level of sentences, paragraphs, and chapters is lost, but analyzing the quantum unit of culture can indicate informative evolutionary trends. We show that pitch classes across five centuries of Western music form a ring (Figs. 23), demonstrating that the foundation of music theory, the circle fifths, is more than a 
metaphor and is a statistical reality. Tonality varies strongly with time (Fig. 4), and the largest source of variance discriminating composers is incremental and linear (Fig. 5), showing that choice of pitch and interval usage among composers is not so much an individualistic choice, but governed by predictable, historical forces. Tonality is predictive of composer identity only to a limited extent but still significantly above chance (Fig. 6), and there are discrete groups of composers with similar tonal styles (Fig. 7). These groups extensively overlap across time (Fig. 5D), at odds with conventional groupings of composers by musical era, but reveal co-existing tonal styles that persist across history.

\section{Materials and Methods}

\section{MIDI (Musical Instrument Digital Interface) files and data processing}

The complete MIDI file databases of Kunst der Fuge (www.kunstderfuge.com) and Classical Archives (www.classicalarchives.com) were downloaded. Additional blues, country, jazz, pop, rock and roll, and ragtime MIDI files were downloaded from The Midkar Group (www.midkar.com) but were not used for analysis. Composer identities associated with each classical MIDI file were noted. Every MIDI file was given an ID number in the form "id_00000" that can be used to track the associated information through subsequent analysis. The ID information can be found in the following GitHub repository: https://github.com/DanChitwood/MIDlocre/

MIDI files were transformed into comma separated data formats using midicsv [15]. The transformed data contained start, end, and pitch values for each note. Track, instrument, and other extractable MIDI data were discarded, as they were not deemed reliable enough for this study. We further processed the data using a customized python script (https://github.com/MIDlocre/analysis/blob/master/analysis3/Processing.py). The extracted data can be found in the following GitHub repository (https://github.com/DanChitwood/MIDlocre). The data is as follows:

Pitch class/key (12 variables): The modulus 12 values for all notes in each bin, summed and scaled for each MIDI file. Denoted "modulated_notes_0-11_withinbins_normalized”.

Within bin analysis (12 variables): The modulus 12 values for all pairwise interval distances within each bin, summed and scaled for each MIDI file. Denoted "modulated_distance_011_withinbin_normalized".

Between bin analysis (12 variables): The modulus 12 values for all pairwise interval distances between successive bins, summed and scaled for each MIDI file. Denoted "modulated_distance_0-11_betweenbin_normalized".

\section{Data analysis}


All code and data needed to recreate the figures in the manuscript and perform the analyses can be found in the following GitHub repository: https://github.com/DanChitwood/MIDlocre

Data analysis was performed in R [24]. Data visualization was performed using ggplot2 [25]. ggrepel was used to visualize text [26] and viridis [27] and ColorBrewer [28] resources for color selection. reshape2, data.table, and plyr [29] were used for formatting data. Principal Component Analysis (PCA) was performed using the prcomp() function. Linear Discriminant Analysis (LDA) was performed using Ida() with the MASS package [30], with CV=TRUE for crossvalidation when predicting composer identities. Bootstrapping for composer identity prediction was performed using a for loop, permuting composer identity against within and between bin interval data, performing an LDA, and recalculating the correct assignment rate over 1,000 iterations. Self-Organizing Map (SOM) analysis was carried out with the kohonen package [31], using a $2 \times 2$ hexagonal grid trained over 10,000 iterations.

\section{References}

1] Rameau JP. Traité de I'harmonie réduite à ses principes naturels. Paris: JBC Ballard; 1722.

2] Huron D. Tone and voice: A derivation of the rules of voice-leading from perceptual principles. Music Perception: An Interdisciplinary Journal. 2001; 19(1):1-64.

3] Diletsky N. Idea grammatiki musikiyskoy. 1679.

4] Heinichen JD. Der General-Bass in der Composition. Dresden; 1728.

5] Euler L. Tentamen novae theoriae musicae ex certissimis harmoniae principiis dilucide expositae. ex typographia Academiae scientiarum. Petropolis: Typogr. Academiae Scientiarvm; 1739.

6] Cohn R. Neo-riemannian operations, parsimonious trichords, and their" tonnetz" representations. Journal of Music Theory. 1997; 41(1):1-66.

7] Tymoczko D. The geometry of musical chords. Science. 2006; 313(5783):72-4.

8] Cooper G, Meyer LB. The rhythmic structure of music. University of Chicago Press; 1963.

9] Kolinski M. A cross-cultural approach to metro-rhythmic patterns. Ethnomusicology. 1973; 494-506.

10] Michel JB, Shen YK, Aiden AP, Veres A, Gray MK, Pickett JP, Hoiberg D, Clancy D, Norvig P, Orwant J, Pinker S, Nowak MA, Aiden EL. Quantitative analysis of culture using millions of digitized books. Science. 2011; 331(6014):176-82. 
11] Bertin-Mahieux T, Ellis DP, Whitman B, Lamere P. The Million Song Dataset. Ismir. 2011; 2(9):10.

12] Soleymani M, Caro MN, Schmidt EM, Sha CY, Yang YH. 1000 songs for emotional analysis of music. In Proceedings of the 2nd ACM International Workshop on Crowdsourcing for Multimedia. 2013; 1-6.

13] Selfridge-Field E, editor. Beyond MIDI: the handbook of musical codes. MIT press; 1997.

14] Good M. MusicXML for notation and analysis. The virtual score: representation, retrieval, restoration. 2001; 12:113-24.

15] Walker J. Midi-CSV; 2008 [cited 2017 Dec 1]. Available from: http://www.fourmilab.ch/webtools/midicsv/

16] Cuthbert MS, Ariza C. music21: A toolkit for computer-aided musicology and symbolic music data. 2010.

17] Bogdanov D, Wack N, Gómez E, Gulati S, Herrera P, Mayor O, Roma G, Salamon J, Zapata JR, Serra X. Essentia: An Audio Analysis Library for Music Information Retrieval. Ismir. 2013; 493498.

18] Giannakopoulos T, Pikrakis A. Introduction to Audio Analysis: A MATLAB ${ }^{\circledR}$ Approach. Academic Press; 2014.

19] McFee B, Raffel C, Liang D, Ellis DP, McVicar M, Battenberg E, Nieto O. librosa: Audio and music signal analysis in python. In Proceedings of the 14th Python in Science Conference 2015; 18-25.

20] Serrà J, Corral Á, Boguñá M, Haro M, Arcos JL. Measuring the evolution of contemporary western popular music. Scientific Reports. 2012; 2:521.

21] Mauch M, MacCallum RM, Levy M, Leroi AM. The evolution of popular music: USA 19602010. Royal Society Open Science. 2015; 2(5):150081.

22] Chitwood $\mathrm{DH}$. Imitation, genetic lineages, and time influenced the morphological evolution of the violin. PloS One. 2014; 9(10):e109229.

23] Park D, Bae A, Schich M, Park J. Topology and evolution of the network of western classical music composers. EPJ Data Science. 2015; 4(1):2.

24] R Core Team. R: A language and environment for statistical computing [Internet]. Vienna, Austria: R Foundation for Statistical Computing; 2017. 
25] Wickham H. ggplot2: elegant graphics for data analysis. Springer; 2016.

26] Slowikowski K. ggrepel: Repulsive Text and Label Geoms for 'ggplot2', 2016.

27] Garnier S. viridis: Matplotlib default color map. R package version 0.2. 2015.

28] Harrower M, Brewer CA. ColorBrewer. org: an online tool for selecting colour schemes for maps. The Cartographic Journal. 2003;40(1):27-37.

29] Wickham H. Tidy data. Journal of Statistical Software. 2014 Aug 1;59(10):1-23.

30] Ripley B, Venables B, Bates DM, Hornik K, Gebhardt A, Firth D, Ripley MB. Package 'MASS'. CRAN Repository. 2013.

31] Wehrens R, Buydens LM. Self-and super-organizing maps in R: the Kohonen package. J Stat Softw. 2007; 21(5):1-9.

\section{Figure Legends}

Figure 1: Creating summaries of pitch class and interval use with bins. A) The first two measures of the Prelude in C major from the Well-Tempered Clavier, Book I (BWV 846). B) For every time point that a note begins or ends in a MIDI file, a new "bin" is created. Shown are the octaves and notes and MIDI note frequency values divided into bins (vertical bars) for every time a note begins or ends for the first two measures of the Prelude in C. C) Modulus 12 is applied to the MIDI note values in B) to arrive at pitch class values for each note, independent of octave. For pitch class/key analysis: Mod12 values, 0-11, for each bin are tallied for the MIDI file and then scaled (mean $=0$, variance $=1$ ). For "within" bin analysis: all pairwise differences (0-11) between Mod12 values within a bin are taken, tallied for the MIDI file, and then scaled. For "between" bin analysis: all pairwise differences (0-11) between Mod12 values between successive bins are taken, tallied for the MIDI file, and then scaled.

Figure 2: Principal Component Analysis (PCA) based on pitch class frequency recreates the circle of fifths. A) For each of $>26,000$ MIDI files, the 12 chromatic pitch classes of notes represented in each bin were tallied and scaled. Plotting PC2 vs. PC1 reveals 12 major densities of MIDI files that correspond to major and minor keys, as shown by projecting major (black) and minor (blue) triads for each key onto the PCA space. B) All pairwise intervals of pitch classes for each key are projected onto the PCA space. The first letter indicates the lower note and the second number the number of chromatic steps away the higher note is. Inverted intervals form concentric rings and are indicated by color: Perfect fifth/perfect fourth (light/dark blue); minor seventh/major second (light/dark green); major sixth/minor third (yellow/light orange); minor sixth/major third (light/dark red); major seventh/minor second (light/dark purple); perfect unison (dark orange); diminished fifth (black). C) Major and D) minor compositions with BachWerke-Verzeichnis (BWV) numbers by Johann Sebastian Bach projected onto the PCA space. Key is indicated by color for each relative major/minor pair. E) Major and F) minor pitch class 
usage for compositions with BWV numbers. The scaled value of note usage for BWV compositions is indicated by color (low to high usage, dark purple to yellow).

Figure 3: Shifts in key across history. A) Year of composer death is projected onto the Principal Component Analysis (PCA) space based on key. Early music compositions tend to occupy regions corresponding to simpler key signatures and the outer edges whereas more modern pieces occupy regions associated with complex key signatures and are more internal. Year of composer death is indicated by color (earlier to later, yellow to black). B) Compositions by example composers are projected onto the PCA space: Giovanni Pierluigi da Palestrina (c. 15251594, black); Antonio Vivaldi (1678-1741, yellow); Richard Wagner (1813-1883, blue); Arnold Schoenberg (1874-1951, magenta); Dmitri Shostakovich (1906-1975, brown).

Figure 4: Principal Component Analysis (PCA) based on interval usage within and between bins. All pairwise intervals A) within each bin and B) between bins were tallied for each MIDI file, scaled, and PCAs performed. PC2 vs. PC1 and PC4 vs. PC3 are plotted for each PCA, with corresponding percent variation for each PC shown. Compositions are colored by year of death of composer (earlier to later, black to yellow).

Figure 5: Interval usage discriminating composer identity correlates with time. A) Percent variance discriminating composers from a Linear Discriminant Analysis (LDA) performed on within and between bin interval usage. LD1 (highlighted in red) explains $14.2 \%$ of the variance. B) The Benjamini-Hochberg adjusted - $\log _{10} p$ value vs. Spearman's rho for each LD correlated with year of composer death. LD1 is uniquely correlated with year of composer death (BHadjusted $p$ value $=1.55 \times 10^{-48}$, rho $=0.82$ ) among $L D$, indicated in red. C) The linear relationship between LD1 (y axis) and composer year of death ( $x$ axis). D) LD1 vs. composer year of death, labeled with composer names. Color indicates groups of composers with similar tonalities, as determined using a Self-Organizing Map (SOM). Orange, Early Renaissance/Early Baroque; Magenta, Renaissance; Lavender, Baroque to Modern; Green, Schoenberg.

Figure 6: Interval usage predicts composer identity. A confusion matrix for composer identity resulting from a Linear Discriminant Analysis (LDA) performed on within and between bin interval usage. Actual composer identity is plotted against predicted composer identity. Composers are arranged in groups with similar tonalities, as determined using a Self-Organizing Map (SOM). The proportion of compositions assigned to each predicted composer falls along each row, and is colored as the square root of the assigned proportion (from low to high, yellow to black). SOM groups are indicated by arrows and color below the matrix. Composers within each group tend to be mistaken for each other at rates higher than for composers outside the group. Orange, Early Renaissance/Early Baroque; Magenta, Renaissance; Lavender, Baroque to Modern; Green, Schoenberg.

Figure 7: Historical shifts in tonality. A heat map, plotting composers against within (w) and between (b) bin interval usage. Intervals have been arranged by hierarchical clustering, so that intervals with similar usage patterns across composers are placed near each other. Each tile is colored by its scaled value, indicating interval usage (low to high, black to yellow). Composers 
are arranged by groups sharing common interval usage patterns determined using a SelfOrganizing Map (SOM). SOM groups are indicated by arrows and color. Orange, Early Renaissance/Early Baroque; Magenta, Renaissance; Lavender, Baroque to Modern; Green, Schoenberg. 


\section{$\mathbf{A}$}

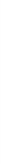

B

Note $\mathrm{MIDI}$

6F 77 6E 76

$6 \mathrm{E} \quad 76 \ldots$

6D $74 \ldots$

6 C $72 \cdots$

5 A $69 \ldots \ldots$

$5 G \quad 67 \ldots$

$5 \mathrm{E} \quad 64 \cdot \rightarrow$

5D $62 \ldots$

$5 C \quad 60$

Bins

Note Mod12
A
$9 \cdot \ldots$
G
G $\quad 7 \cdots$
$\mathrm{F}$
E
$5 \ldots \ldots$
E $\quad 4 \cdot-$
D $2 \ldots \ldots$
C
$\mathrm{C} \quad 0 \rightarrow$
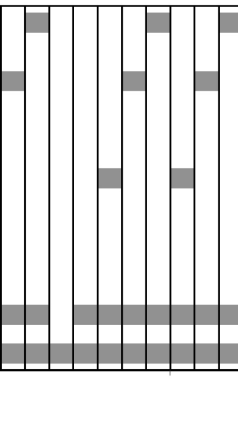

$\rightarrow$ 
\begin{tabular}{l|l|} 
A & - Major
\end{tabular}

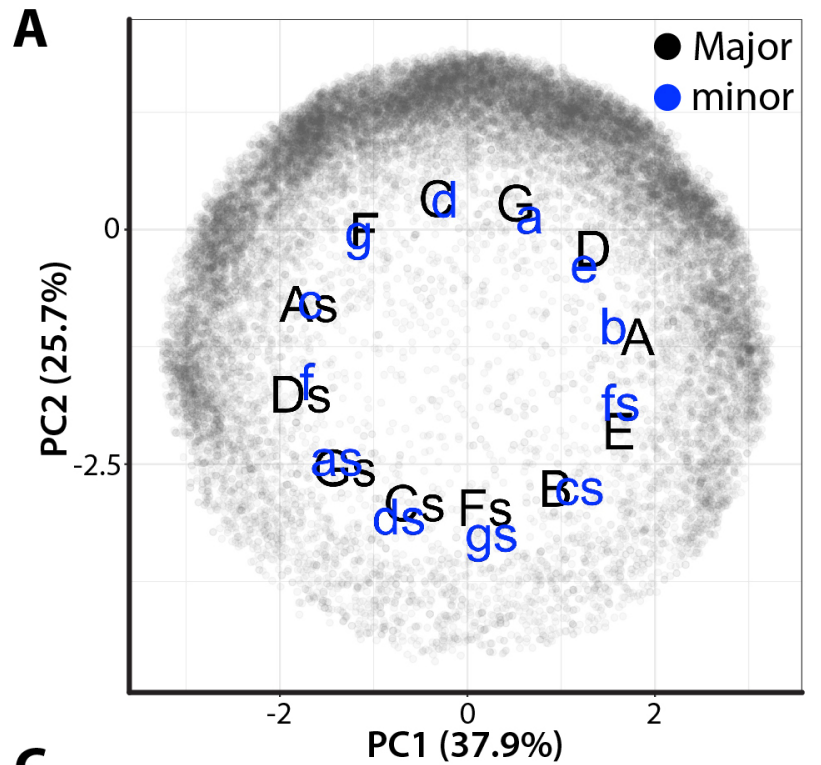

C

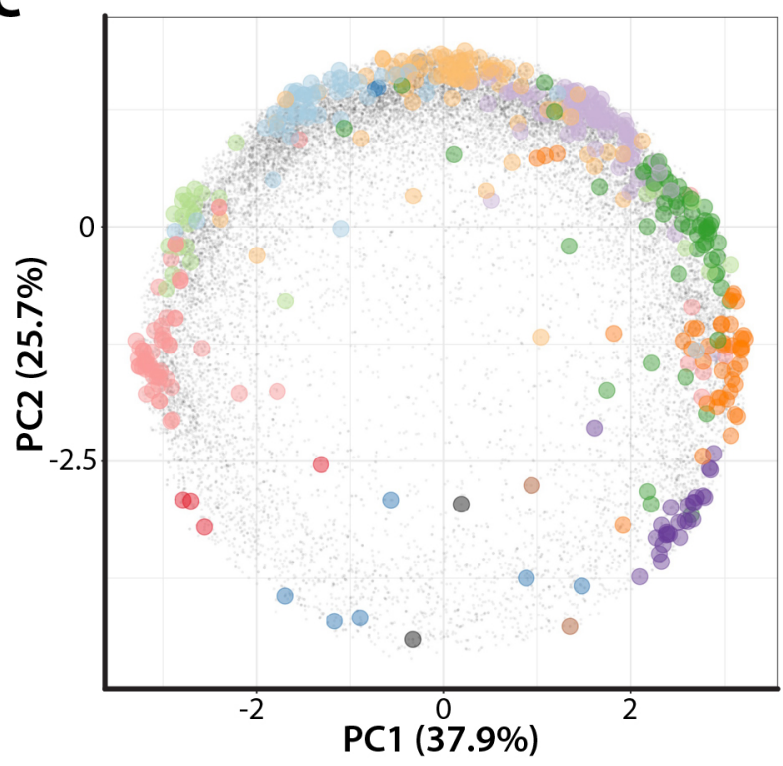

E

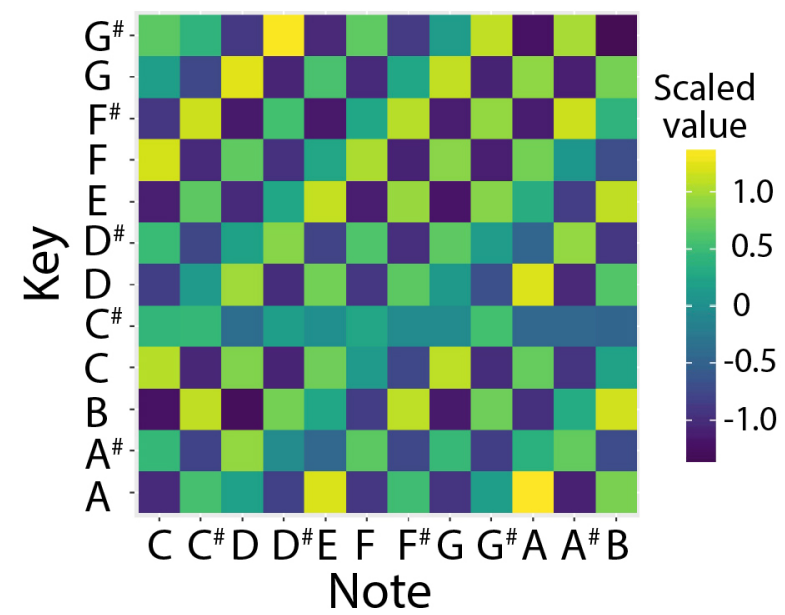

B

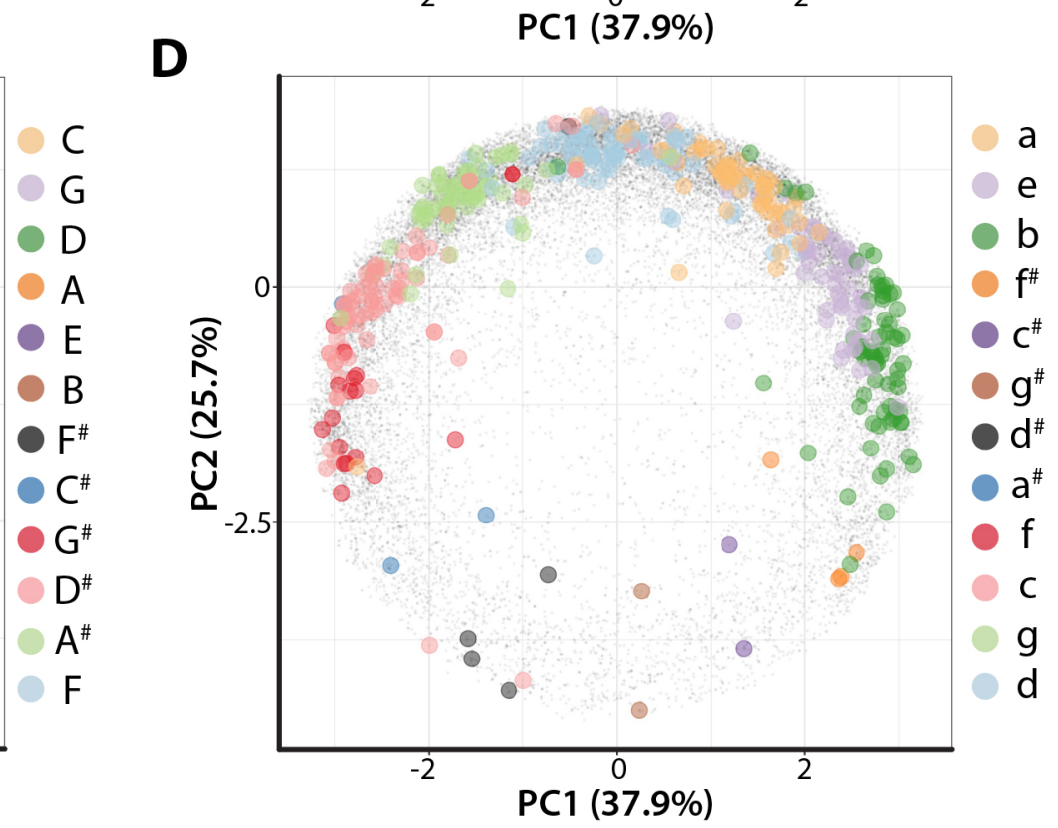

F

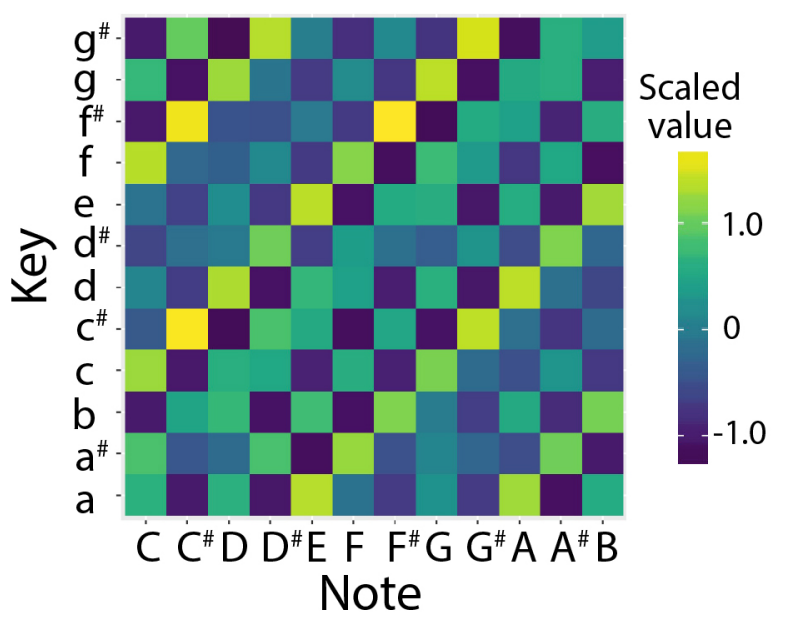

P5

P4

$\mathrm{m} 7$

$\mathrm{M} 2$

M6

m3

P1

$\mathrm{m} 6$

M3

M7

$\mathrm{m} 2$

d5 Note 


\section{A}
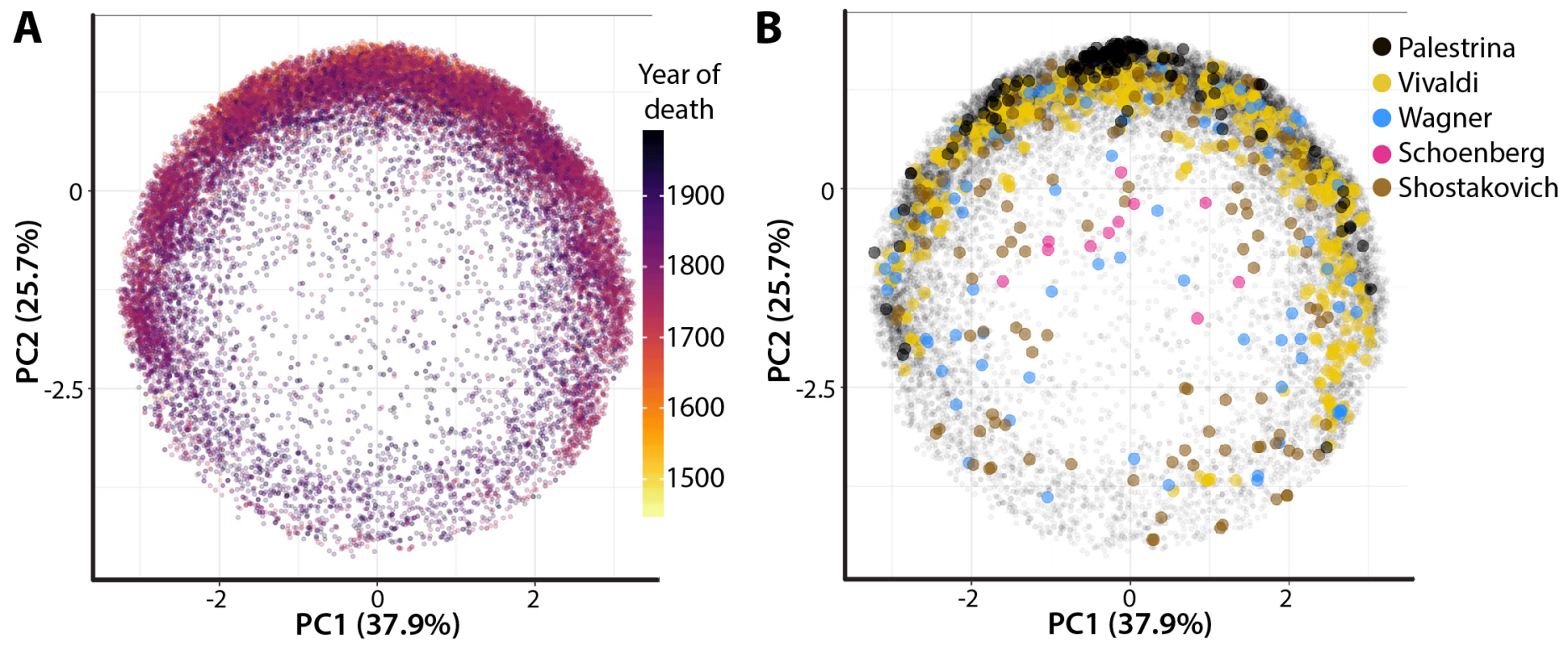


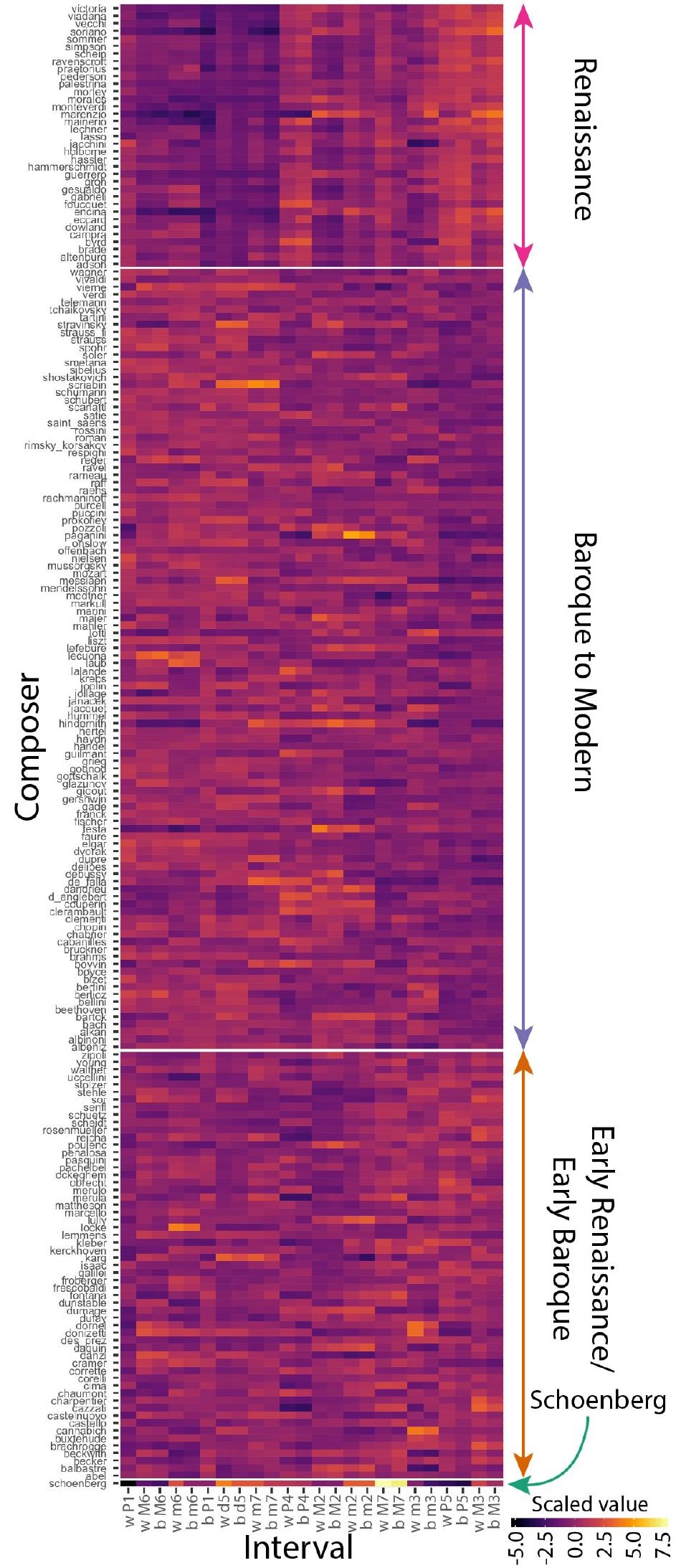

